\title{
e-LORS: Uma Abordagem para Recomendação de Objetos de Aprendizagem
}

\author{
Title: e-LORS: Learning Objects Recommendation Approach
}

\author{
Luciana A M Zaina \\ Universidade Federal de São \\ Carlos, campus Sorocaba \\ lzaina@ufscar.br
}

\author{
Graça Bressan \\ Universidade de São Paulo \\ (PCS - POLI - USP) \\ gbressan@larc.usp.br
}

\author{
Maria A C A Cardieri \\ Faculdade de Engenharia \\ de Sorocaba \\ angelicacc@facens.br
}

\author{
José F Rodrigues Jr \\ Universidade de São Paulo \\ (ICMC-USP) \\ junio@icmc.usp.br
}

\begin{abstract}
Resumo $O$ uso de sistemas de recomendação com a finalidade de melhorar a interação usuáriocomputador tem crescido em aplicações eletrônicas como e-commerce e e-learning. Sistemas de recomendação visam sugerir informações e serviços que possam ser atrativos baseando-se no uso automatizado de dados que descrevem as preferências do usuário. Na área educacional, a recomendação de conteúdos mais relevantes pode atrair a atenção do estudante, motivando-o durante o processo de ensino-aprendizagem. Uma das formas de se alcançar este objetivo se dá por meio do processamento computacional de dados que descrevem o estilo de aprendizagem do estudante. Neste contexto, o objetivo deste trabalho é apresentar a metodologia e-LORS, uma abordagem para a recomendação de conteúdo eletrônico baseada no relacionamento entre perfis e objetos de aprendizagem. Na metodologia proposta, perfis de aprendizagem são descritos por dimensões discretas de maneira a atender diferentes perspectivas de preferência do estudante. Já a metodologia de recomendação usa estas dimensões para filtrar os objetos de aprendizagem - descritos pelo padrão IEEE LOM - mais adequados ao estudante. Palavras-Chave: aprendizagem eletrônica, objeto de aprendizagem, perfil de aprendizagem, sistemas de recomendação, padrão IEEE LOM
\end{abstract}

\begin{abstract}
The use of recommender systems in order to improve human-computer interaction has increased significantly in applications as e-commerce and e-learning. Such systems aim at suggesting information and services that can be attractive to the users based on the automatic use of data that describe their preferences. In the educational area, the recommendation of relevant and interesting contents can be effective for catching the students' attention and driving higher motivation during the learning-teaching process. The ability to provide contents of their preferences considering the students learning style may increase substantially the success of such effort. In this context, the goal of this work is to present the e-LORS system, an approach for recommendation of electronic content based on the relationship between learning styles and learning objects. In our methodology, the learning profiles are described according to discrete dimensions in order to support different perspectives concerning the students' preferences. Our recommendation methodology, in turn, uses these dimensions to filter the learning objects - described by the IEEE LOM standard - that are more suitable to the students.
\end{abstract}

Keywords: e-learning, learning object, learning profile, recommendation system, IEEE LOM standard 


\section{Introdução}

A personalização de ambientes de aprendizagem eletrônica de acordo com as preferências do aprendiz tem sido um tema amplamente estudado. O principal objetivo dessa personalização é prover o aluno com serviços e informações que sejam relevantes ao processo do ensinoaprendizagem e que ao mesmo tempo atendam ao seu perfil.

A observação do estilo de aprendizagem do aluno é uma das maneiras de se identificar características que sejam relevantes para a adequada recomendação de conteúdo. Um estilo de aprendizagem pode determinar como um aluno interage e reage em um ambiente de aprendizagem eletrônica (e-learning) refletindo suas preferências reais. Em síntese, estilos de aprendizado incorporam as características individuais de um aluno referentes às tarefas de organizar, perceber, processar, lembrar e pensar para resolver um problema.

Para que as informações sobre o estilo do aluno possam ser empregadas de forma sistemática em um ambiente eletrônico é necessário um modelo que auxilie o processo. A criação de um tal modelo dá suporte à organização das informações que descrevem o aluno. Este tipo de modelagem tem sido um importante componente dentro do processo de adaptação de ambientes de aprendizagem eletrônica; ele permite que o sistema direcione as ações a serem realizadas de acordo com a interação do aluno, sobretudo o oferecimento de conteúdos eletrônicos mais adequados.

Os conteúdos exibidos ao aluno podem ser organizados de diversas maneiras; uma delas é por meio da catalogação de metadados que descrevam estes conteúdos como objetos de aprendizagem (learning objects). Os metadados permitem automatizar a busca e a recuperação de conteúdos e, ao seguirem um padrão bem estabelecido, podem ser reutilizados em diferentes escopos de aprendizagem.

Neste cenário, este trabalho tem como objetivo apresentar a metodologia e-LORS (e-Learning Object Recommendation System - Sistema de Recomendação de Objetos de Aprendizagem Eletrônica), uma abordagem para recomendação de conteúdo eletrônico baseada no relacionamento entre perfis e objetos de aprendizagem. $\mathrm{Na}$ metodologia e-LORS, um estilo de aprendizagem é organizado de acordo com dimensões que incorporam as preferências de aprendizagem de um aluno. Seu objetivo é verificar o relacionamento entre as dimensões e os objetos de aprendizagem processando-se os metadados que os descrevem. Torna-se possível, então, a recomendação dos objetos de aprendizagem que mais bem correspondem ao perfil de aprendizagem de um determinado aluno. Para a tarefa de descrição dos perfis de aprendizagem em dimensões discretas, foi adotado o Modelo de Estilos de Aprendizagem de Felder e Silverman [29].

Para validação da abordagem apresentada neste artigo foi realizado um experimento com alunos dos cursos de Engenharia Civil, Engenharia da Computação e Engenharia Elétrica em turmas da disciplina de Física I durante todo um semestre letivo.

O restante do artigo está estruturado da seguinte forma: a seção 2 discute conceitos sobre perfil de aprendizagem, estilos de aprendizagem e objetos de aprendizagem; a abordagem proposta é apresentada na seção 3; a seção 4 descreve a validação da proposta bem como seus resultados; trabalhos relacionados à abordagem são apresentados na seção 5 e a seção 6 realiza as considerações finais discorrendo sobre trabalhos futuros.

\section{Perfil, Estilo e Objetos de Aprendi- zagem}

Os conceitos referentes a perfil, estilo e objeto de aprendizagem são fundamentos importantes da proposta deste trabalho, como descritos nas próximas seções.

\subsection{Perfil de Aprendizagem}

Nos ambientes de aprendizagem eletrônica é fundamental a obtenção de informações sobre os perfis dos alunos para a personalização automática do ambiente em relação às necessidades dos alunos. O perfil do aluno é determinado por meio de elementos discretos; dados que permitem ressaltar suas características, possibilitando que ele seja delineado. Seu propósito é organizar as informações de maneira que estas possam ser interpretadas por um ambiente eletrônico [3][27]. Exemplos das características que definem um perfil são: sua identificação pessoal, suas preferências pessoais e sociais, seu conhecimento sobre determinados assuntos, e seu estilo de aprendizagem [15][27]. Em especial, o estilo de aprendizagem é refletido em ações realizadas por um aluno a partir dos estímulos recebidos do ambiente em que está interagindo.

\subsection{Estilo de Aprendizagem}

Um estilo de aprendizagem está relacionado às estratégias que um aluno tende a aplicar com freqüência a uma dada situação de ensino. Cada indivíduo pode se encaixar em diferentes estilos que fazem com que ele adote atitudes e comportamentos que se repetem em diferentes momentos e situações [29][30]. Para satisfazer a diferentes estilos é necessário que o docente utilize estratégias de ensino 
adequadas às várias perspectivas de aprendizagem.

A adoção de estilos de aprendizagem específicos a cada perfil permite que o docente organize o ambiente com mecanismos que trabalham as diferentes habilidades dos alunos, melhorando a percepção para os elementos que são os focos do processo. Isto ocorre pois serão oferecidos a esses alunos situações e elementos que este- jam em consonância com suas necessidades e preferências [7][16][30].

Existem diversos modelos que descrevem modos de classificar um aluno em um determinado estilo de aprendizagem [12][30]. A Tabela 1 apresenta os principais modelos, bem como a principal característica de cada um com exemplos de trabalhos que os aplicaram.

\begin{tabular}{|c|c|c|c|}
\hline $\begin{array}{l}\text { Modelo de } \\
\text { Estilo de } \\
\text { Aprendizagem }\end{array}$ & Dimensões & Característica Principal & Exemplos de Aplicação do Modelo \\
\hline $\begin{array}{l}\text { Myers-Briggs } \\
\text { Type Indicator } \\
\text { (MBTI) }\end{array}$ & $\begin{array}{l}\text { - Extrovertido/Introvertido } \\
\text { - Sensorial/Intuitivo } \\
\text { - Racionais/Emocionais } \\
\text { - Julgadores/ Perceptivos }\end{array}$ & $\begin{array}{l}\text { Para cada dimensão há uma } \\
\text { característica dominante. }\end{array}$ & $\begin{array}{l}\text { Soles e Moller [9] apresentam um sistema de aprendiza- } \\
\text { gem eletrônica que disponibiliza atividades (materiais e } \\
\text { ferramentas) de acordo com o modelo de Myers-Briggs. } \\
\text { Salim e Haro [25] aplicam conceitos de lógica nebulosa } \\
\text { (fuzzy) para classificação dos alunos nos estilos de } \\
\text { aprendizagem de Myers-Briggs. }\end{array}$ \\
\hline $\begin{array}{l}\text { Kolb's Experien- } \\
\text { tial Learning } \\
\text { Model }\end{array}$ & $\begin{array}{l}\text { - Não Focado (Diverger) = Concreto + Reflexivo } \\
\text { - Assimilador (Assimilator) = Abstrato + Reflexivo } \\
\text { - Focado (Converger) = Abstrato + Ativo } \\
\text { - Acomodador (Accomodator) = Concreto + Ativo }\end{array}$ & $\begin{array}{l}\text { O estudante possui uma dimen- } \\
\text { são que se sobressai sobre as } \\
\text { demais. }\end{array}$ & $\begin{array}{l}\text { Uma experiência utilizando o modelo de Kolb para acom- } \\
\text { panhar os alunos através de duas situações: uma com } \\
\text { aprendizagem online, outra com aprendizagem tradicional, } \\
\text { foi apresentada por Lu et al. [17]. }\end{array}$ \\
\hline $\begin{array}{l}\text { Honey and } \\
\text { Mumford's } \\
\text { Learning Styles } \\
\text { Questionnaire } \\
\text { (LSQ) }\end{array}$ & $\begin{array}{l}\text { - Ativistas } \\
\text { - Reflexivos } \\
\text { - Teóricos } \\
\text { - Pragmáticos }\end{array}$ & $\begin{array}{l}\text { Resume o modelo de Kolb em } \\
\text { quatro dimensões bem defini- } \\
\text { das. }\end{array}$ & $\begin{array}{l}\text { A plataforma AHA! apresentada por Stash et al. [26] } \\
\text { apresenta uma ferramenta de autoria que permite que } \\
\text { sejam relacionados, manualmente, os estilos de aprendi- } \\
\text { zagem aos materiais durante sua catalogação. } \\
\text { Um experimento realizado com alunos de engenharia } \\
\text { elétrica foi apresentado por Lowery [7]. A autora retrata o } \\
\text { uso do modelo de perfis como meio de determinar os } \\
\text { melhores mecanismos de ensino-aprendizagem a serem } \\
\text { adotados pelos estudantes. }\end{array}$ \\
\hline $\begin{array}{l}\text { Herrmann Brain } \\
\text { Dominance } \\
\text { Instrument }\end{array}$ & $\begin{array}{l}\text { - Teórico } \\
\text { - Organizador } \\
\text { - Inovador } \\
\text { - Humanitário }\end{array}$ & $\begin{array}{l}\text { O hemisfério do cérebro que é } \\
\text { dominante é influenciado } \\
\text { socialmente (ensino, experiência } \\
\text { de vida, influências culturais) e } \\
\text { não geneticamente. }\end{array}$ & $\begin{array}{l}\text { Uma experiência com a utilização de tecnologias para } \\
\text { aprendizagem empregando } 0 \text { modelo foi realizada por } \\
\text { Lumsdaine et al. [11]. }\end{array}$ \\
\hline $\begin{array}{l}\text { Felder-Silverman } \\
\text { Learning Style } \\
\text { Model }\end{array}$ & $\begin{array}{l}\text { - Sensorial//ntuitivo } \\
\text { - } \text { Visual/Verbal } \\
\text { - Stivo/Reflexivo } \\
\text { - Sequencial/Global }\end{array}$ & $\begin{array}{l}\text { Existe um elemento dominante } \\
\text { para cada dimensão. }\end{array}$ & $\begin{array}{l}\text { Uma metodologia para identificação de estilos de aprendi- } \\
\text { zagem por meio de padrões de comportamento observa- } \\
\text { dos em Sistemas Gerenciadores de Aprendizagem (Lear- } \\
\text { ning Management Systems - LMS) foi proposto por Graf et } \\
\text { al. [32]. A abordagem emprega o modelo de Felder para } \\
\text { identificação dos perfis a partir dos padrões. } \\
\text { Outro trabalho Lazzarotto et al, [40] utiliza a proposta de } \\
\text { Felder e Silverman para classificar os alunos em } 4 \text { grupos } \\
\text { distintos considerando as dimensões intuitiva/sensitiva e } \\
\text { global/sequencial. }\end{array}$ \\
\hline
\end{tabular}

Tabela 1: Modelos de estilos de aprendizagem, suas dimensões e característica principal, e trabalhos que aplicam estes estilos.

O modelo MBTI descreve que as preferências individuais estão diretamente conectadas com a personalidade do indivíduo. Ele apresenta os estilos individuais em quatro escalas com classificações antagônicas para cada uma delas, prevendo 16 tipos distintos determinados a partir das possíveis combinações [9]. Por exemplo, um indivíduo pode ter os valores Extrovertido - Racional Pensador - Atento.

O modelo de Kolb (Kolb's Learning Style Inventory) foca na transformação do conhecimento que é observado a partir da experiência do aluno adquirida no processo de aprendizagem. As escolhas sobre como o estudante prefere receber a informação e como deseja processá-la indicam seu estilo [2].

Honey e Mumford apresentam uma simplificação do modelo de Kolb através de um questionário que indica os pontos fortes e fracos para cada dimensão do perfil do estudante [14].
Identificar a inteligência dominante do estudante por meio da aplicação de uma auto-avaliação é o objetivo do modelo de Herrmann (Herrmann Brain Dominance Instrument model). As preferências individuais são definidas por meio da identificação de qual hemisfério do cérebro tem influência no processo de aprendizagem [13].

O modelo de Felder-Silverman descreve o estilo de aprendizagem por meio de 4 dimensões. O princípio deste trabalho é a associação entre estilos de aprendizagem e estratégias de ensino mais adequadas [29][33]. Este modelo é indicado para cursos na área de engenharia [30]. A Tabela 2 detalha o modelo de Felder-Silverman. Além de estar associada a um estilo de aprendizagem, cada dimensão do modelo também se relaciona com os métodos de ensino que podem ser aplicados. Desta maneira, a associação entre o estilo de aprendizagem e o método de ensino possibilita que o sistema eletrônico ofereça os materiais mais adequados às preferências de aprendizagem [33]. 


\begin{tabular}{|c|c|c|c|}
\hline Dimensão & Características & $\begin{array}{l}\text { Estilo de } \\
\text { Aprendiza- } \\
\text { gem }\end{array}$ & $\begin{array}{c}\text { Método de } \\
\text { Ensino }\end{array}$ \\
\hline \multirow{2}{*}{ Percepção } & \multirow{2}{*}{$\begin{array}{l}\text { Está relacionada a como o } \\
\text { aluno recebe o conteúdo; tipos } \\
\text { de exercícios, por exemplo. }\end{array}$} & Sensorial & Concreto \\
\hline & & Intuitivo & Abstrato \\
\hline \multirow{2}{*}{$\begin{array}{l}\text { Formato- } \\
\text { Apresenta- } \\
\text { ção }\end{array}$} & \multirow{2}{*}{$\begin{array}{l}\text { Está relacionado à forma como } \\
\text { o conteúdo é apresentado; } \\
\text { tipos de mídias, por exemplo. }\end{array}$} & Visual & Visual \\
\hline & & Auditivo & Verbal \\
\hline \multirow{2}{*}{$\begin{array}{l}\text { Participação } \\
\text { do Aluno }\end{array}$} & \multirow{2}{*}{$\begin{array}{l}\text { Representa o quanto o aluno } \\
\text { gosta de participar das ativida- } \\
\text { des; por exemplo, se possui } \\
\text { liderança, ou se prefere refletir } \\
\text { mais tempo sobre um dado } \\
\text { assunto. }\end{array}$} & Ativo & Ativo \\
\hline & & Reflexivo & Passivo \\
\hline \multirow{2}{*}{ Sequência } & \multirow{2}{*}{$\begin{array}{l}\text { Determina como deve ser a } \\
\text { ordem de apresentação de um } \\
\text { conteúdo. } \\
\text { O aluno sequencial prefere que } \\
\text { as informações sejam apresen- } \\
\text { tadas de forma progressiva. Já } \\
\text { o global prefere ter uma visão } \\
\text { do todo, dos objetivos, para } \\
\text { então visualizar as partes. }\end{array}$} & Sequencial & Progressivo \\
\hline & & Global & Visão Geral \\
\hline
\end{tabular}

Tabela 2: Dimensões baseadas no modelo de Felder e Silveman [29].

\subsection{Objetos de Aprendizagem}

Um dos objetivos de um ambiente de ensinoaprendizagem é oferecer materiais didáticos, usualmente denominados de objetos de aprendizagem (LO - Learning Objects), que sejam aderentes ao perfil de aprendizagem do aluno.

Um objeto de aprendizagem pode ser definido como uma entidade a ser utilizada dentro do processo de ensino-aprendizagem. Dentre outras coisas, citam-se vídeos, figuras, e/ou simuladores. Dentro do escopo de aprendizagem eletrônica o que se deseja é criar conteúdos em formato digital que possam ser reutilizáveis em diferentes objetivos de aprendizagem; ou mesmo, que possam ser empregados na construção de outros objetos de aprendizagem [19][31].

Uma das formas de organizar os objetos de aprendizagem para que eles possam ser reutilizados e empregados sistemicamente é por meio do uso de metadados [4]. Um metadado é definido como um conjunto de dados que contextualiza as características de um determinado elemento de informação, no caso objetos de aprendizagem [36]. O padrão LOM (Learning Object Metadata) do instituto IEEE (Institute of Electrical and Electronics Engineers) é um padrão que descreve objetos de aprendizagem segundo um padrão universalmente aceito, o que facilita a interoperabilidade entre diferentes repositórios que o empregam [19][21].

\section{Sistema de Recomendação de Obje- tos de Aprendizagem}

Os sistemas de recomendação têm sido adotados em di- versas áreas, como em comércio eletrônico, sistemas de museus, aprendizagem eletrônica, entre outros; sendo que seu objetivo é prover o usuário com informações e serviços que estejam em consonância com seus interesses.

As recomendações são realizadas tendo-se como base características tanto do sistema quanto dos interesses do usuário. Assim, um sistema de recomendação no contexto de aprendizagem sugere materiais e serviços de acordo com as ações que o aluno executa [1][8].

\subsection{Algoritmos de filtragem}

Para proceder com a escolha de conteúdo, algoritmos de filtragem da informação baseados no perfil do usuário são aplicados pelos sistemas de recomendação [22]. Tais algoritmos utilizam a modelagem do usuário como orientação, pois esta modelagem incorpora os diferentes perfis de aprendizagem. Além disto, tais algoritmos devem considerar o tipo de sistema em que está sendo empregado, de maneira a atender as características de interação presentes [35]. Duas técnicas empregadas na área de filtragem de informação podem ser destacadas: a colaborativa e a baseada em conteúdo. Na abordagem colaborativa a informação é filtrada com base no que é relevante a outros usuários que possuem hábitos similares ao usuário em questão. Já na baseada em conteúdo, a filtragem é realizada por meio da correlação entre o conteúdo e as preferências do usuário [28][35].

\subsection{O sistema e-LORS}

Especificamente, o modelo proposto neste artigo, denominado de e-LORS (e-Learning Object Recommendation System), se baseia nos conceitos empregados nos sistemas de recomendação, utilizando um algoritmo de filtragem da informação baseada em conteúdo [23][37].

A arquitetura e-LORS foi especificada de maneira que possa ser utilizada por diferentes sistemas de aprendizagem eletrônica. A recomendação de objetos é realizada confrontando-se as informações do tema de estudo a ser apresentado ao aluno, o perfil de aprendizagem do aluno, e as possíveis restrições tecnológicas que caracterizam o ambiente eletrônico em que o aluno interage. Estas informações são obtidas pelo sistema e-LORS por meio do modelo do aluno e do gerenciador de cursos. A Figura 1 apresenta a arquitetura e-LORS e sua integração com um sistema de aprendizagem eletrônica.

As subseções a seguir apresentam a proposta de divisão de preferências de aprendizagem em dimensões, os requisitos necessários para adoção do e-LORS e o detalhamento das filtragens executadas. 


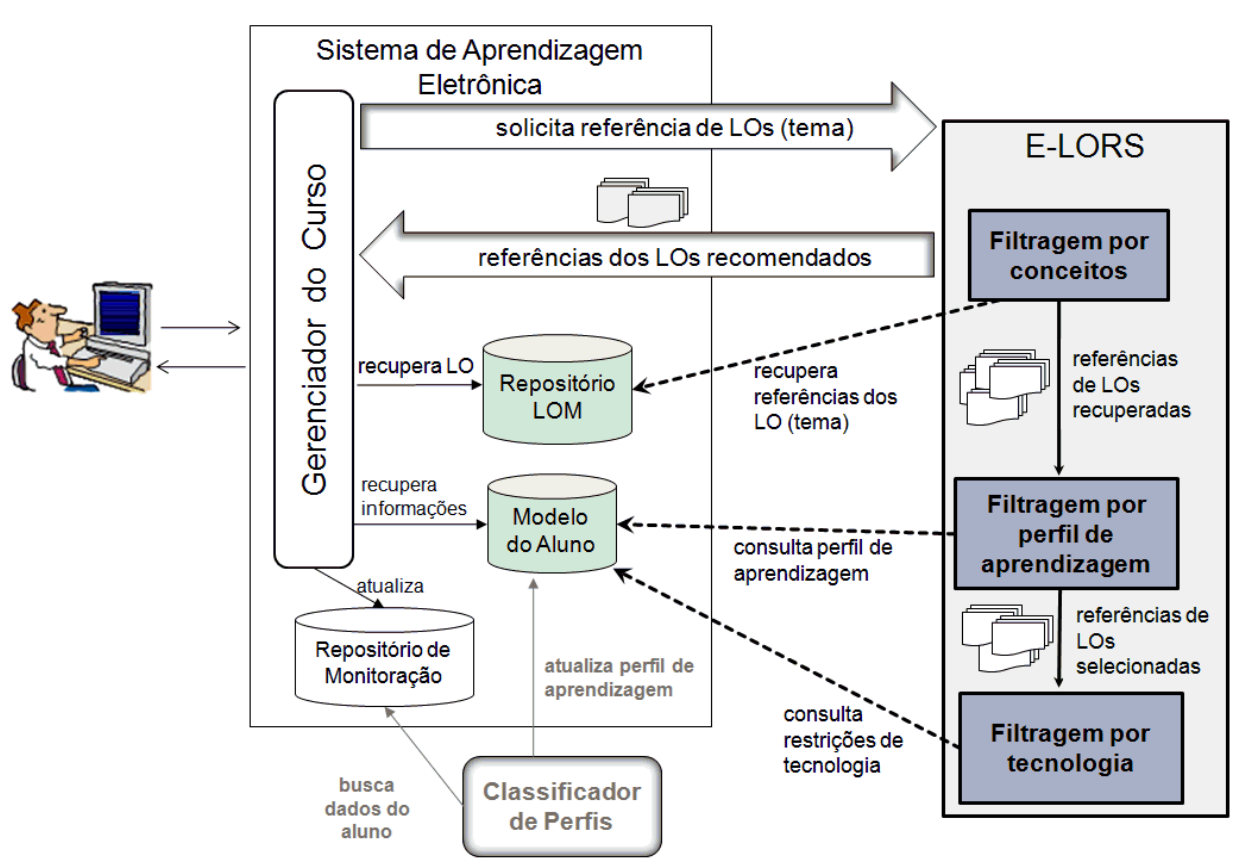

Figura 1: Arquitetura do sistema e-LORS e sua integração com um sistema de aprendizagem eletrônica.

\subsection{Dimensões de Preferências}

A confrontação de informações particulares sobre um determinado aluno com os requisitos necessários para a recomendação são fundamentais para a recomendação de conteúdo [39]. Para este propósito, é necessário um modelo de aluno muito bem definido, contendo múltiplas informações, e sendo elaborado de acordo com as necessidades que um determinado sistema tem para automatizar suas tarefas [8].

\begin{tabular}{|c|c|c|c|}
\hline Componente & \multicolumn{2}{|c|}{ Atributos } & Caracterização \\
\hline $\begin{array}{l}\text { Informações } \\
\text { Pessoais }\end{array}$ & \multicolumn{2}{|c|}{$\begin{array}{ll}\circ & \text { Identificação no sistema } \\
\circ & \text { Nome } \\
\circ & \text { Grau de escolaridade } \\
\circ & \text { Curso de formação }\end{array}$} & $\begin{array}{l}\text { Os dados pessoais se caracterizam por conter as informações pessoais do aluno, } \\
\text { as quais raramente são alteradas. }\end{array}$ \\
\hline \multirow{3}{*}{$\begin{array}{c}\text { Perfil de } \\
\text { aprendizagem }\end{array}$} & \multirow{3}{*}{ Preferências } & Percepção & \multirow{3}{*}{$\begin{array}{l}\text { O perfil de aprendizagem é definido em categorias de preferências, que permitem } \\
\text { visualizar o perfil de aprendizagem do estudante em diferentes dimensões. Esta } \\
\text { dimensão é o coração do sistema de recomendação. }\end{array}$} \\
\hline & & Formato-Apresentação & \\
\hline & & Participação do Aluno & \\
\hline Tecnologia & \multicolumn{2}{|c|}{ Restrições Tecnológicas } & $\begin{array}{l}\text { Esta informação deve ser obtida por meio da intervenção direta do aluno permitin- } \\
\text { do que sejam disponibilizados recursos mais adequados a ele. São exemplos de } \\
\text { restrições: tipo de equipamento, conexão de rede, sistema operacional utilizado, } \\
\text { entre outras. Este é um elemento opcional no modelo do aluno. }\end{array}$ \\
\hline
\end{tabular}

Tabela 3: Modelo do aluno com as dimensões de preferências.

O modelo do aluno adotado neste trabalho divide as preferências do estudante em dimensões que são baseadas na proposta de Felder e Silverman (Tabela 2) [29]. As dimensões utilizadas são Percepção, FormatoApresentação e Participação do Aluno. Para a presente proposta não foi adotada a dimensão Sequência, pois o conteúdo usado nos experimentos não abrangeria a complexidade desta dimensão. Além das dimensões de Felder e Silverman, o modelo apresenta informações pessoais do aluno, e características tecnológicas relacionado ao acesso do aluno.

A Tabela 3 apresenta o modelo destacando os compo- 
nentes que influenciam na caracterização do perfil de aprendizagem. Nesta concepção, as dimensões de preferências irão refletir o comportamento do aluno segundo diferentes perspectivas [20][21]. Uma das vantagens da proposta é que a subdivisão em dimensões permite que o sistema possa atender diversas perspectivas de preferências distintas.

\subsection{Requisitos para Adoção do e-LORS}

O modelo e-LORS foi elaborado para ser adotado em diferentes arquiteturas de sistemas de aprendizagem eletrônica, para o quê, alguns requisitos devem ser atendidos.

\subsubsection{Padrão LOM}

Um requisito importante é a armazenagem e o gerenciamento dos objetos de aprendizagem utilizando o padrão LOM [19]. Assim, a recomendação irá associar os objetos descritos pelo padrão com as categorias de perfil. Embora o padrão apresente um grande número de campos de especificação, o sistema e-LORS utilizará apenas alguns. A Tabela 4 apresenta as categorias LOM utilizadas e seus respectivos campos.

\begin{tabular}{|c|l|l|}
\hline $\begin{array}{c}\text { Categoria } \\
\text { LOM }\end{array}$ & \multicolumn{1}{|c|}{ Campos do LOM } & Caracterização \\
\hline Geral & $\begin{array}{l}\text { Identificador; Tipo; Título; Língua; } \\
\text { Descrição e Palavras-chaves. }\end{array}$ & $\begin{array}{l}\text { Faz a descrição geral do } \\
\text { objeto de aprendizagem. }\end{array}$ \\
\hline Técnico & $\begin{array}{l}\text { Formato: como vídeo, ou som, por } \\
\text { exemplo; Tamanho digital; Locali- } \\
\text { zação física e Requisitos para uso } \\
\text { do objeto; por exemplo, versão de } \\
\text { software necessário ao formato. }\end{array}$ & $\begin{array}{l}\text { Descrição de caracterís- } \\
\text { ticas técnicas. }\end{array}$ \\
\hline $\begin{array}{c}\text { Educacio- } \\
\text { nal }\end{array}$ & $\begin{array}{l}\text { Tipo de Interatividade (ativo, } \\
\text { expositivo) e Tipo de Recurso da } \\
\text { Aprendizagem (exercício, simula- } \\
\text { ção, questionário) e Grau de } \\
\text { dificuldade. }\end{array}$ & $\begin{array}{l}\text { Descrição das funções } \\
\text { educacionais e caracte- } \\
\text { rísticas pedagógicas do } \\
\text { objeto. }\end{array}$ \\
\hline $\begin{array}{c}\text { Relaciona- } \\
\text { mento }\end{array}$ & $\begin{array}{l}\text { Tipo de relacionamento entre os } \\
\text { objetos e identificação do relacio- } \\
\text { namento. }\end{array}$ & $\begin{array}{l}\text { Descreve o relaciona- } \\
\text { mento entre obje-tos de } \\
\text { aprendizagem. }\end{array}$ \\
\hline
\end{tabular}

Tabela 4: Categorias descritoras do LOM utilizadas pelo e-LORS [19].

\subsubsection{Modelo do Aluno}

Outro requisito é a adoção de um modelo do aluno que descreva o perfil de aprendizagem usando dimensões de preferências baseadas em Felder e Silverman [30].

Se o sistema de aprendizagem já adotar algum modelo do aluno é preciso que esse modelo seja estendido agregando os elementos de perfil usados no sistema e-LORS.

\subsubsection{Determinação de preferências de aprendi-} zagem
Existem diferentes estratégias para delineação das preferências de aprendizagem de cada aluno. Uma Possibilidade é o uso de questionários respondidos diretamente pelo aluno, o qual irá expressar suas preferências de acordo com cada dimensão. As dimensões seriam atualizadas a partir das respostas dadas pelo aluno.

Uma outra maneira é a adoção de um sistema classificador de perfis como proposto por Zaina e Bressan [20]. Usando-se a monitoração das interações do estudante fazse, então, a comparação de suas atividades com modelos de perfis previamente elaborados. Após um período de observação o classificador de perfis compara o comportamento observado com os modelos de perfil, determinando para cada dimensão de preferência a classificação do aluno. A Figura 1 - parte central inferior - apresenta a possibilidade de inserção do classificador como forma de identificar as preferências do aprendiz.

\subsubsection{Restrição Tecnológica}

Outro ponto é a utilização do item que descreve a tecnologia no modelo do aluno, porém esta característica é opcional. Caso o elemento de restrição tecnológica não seja utilizado, ele pode ser agregado em uma eventual nova implementação. Do contrário, o sistema e-LORS irá desconsiderar esta verificação.

\subsection{Filtragens para Recomendação}

O sistema e-LORS possui, ainda, uma interface de software que define o que deve ser implementado para especificação do processo de recomendação. Nesta interface, o método que dá início ao processo é denominado de recomendation $L O$, o qual possui como parâmetro o tema relacionado aos LOs que serão recomendados e a identificação do aluno que está sendo alvo da recomendação. A partir da chamada deste método, o sistema e-LORS inicia o processo de recomendação retornando ao gerenciador de cursos um conjunto de objetos selecionados com a identificação dos LOs (campo identificador da Categoria Geral LOM) que foram recomendados. O gerenciador usa este conjunto para recuperar os objetos correspondentes e construir a apresentação ao aluno. $\mathrm{O}$ gerenciador de aprendizagem é livre para construir a visualização da forma mais adequada. Ou seja, o e-LORS apenas recupera a identificação dos objetos de aprendizagem, cabendo ao ambiente que o está utilizando construir a visualização do material de ensino.

Após iniciado o processo de recomendação por meio do método recomendation $L O$, a execução segue ao longo de três fases de filtragem, sendo que cada uma é focada em uma dimensão diferente, conforme detalhado a seguir. 


\subsubsection{Filtragem por Conceitos}

A filtragem por conceitos permite recuperar as referências dos objetos de aprendizagem que estejam de acordo com o tema que está sendo buscado no momento.

Assim que o sistema recebe a requisição para a execução do processo de recomendação, ele inicia a filtragem por conceitos, buscando as referências dos LOs em um repositório LOM (LOMR - LOM Repository). A filtragem por conceitos é baseada no tema, que pode ser composto por uma ou mais palavras chaves passadas como parâmetros. Por meio da Categoria Geral, o método search $L O$, busca quais objetos de aprendizagem possuem a ocorrência do tema comparando os campos Título, Descrição e Palavras-chaves, conforme formalizado na equação (1) e no predicado (2). Como resultado será obtido um conjunto de referências LO (LOSet) que satisfazem ao tema.

$$
\begin{aligned}
& \text { searchLO: LOMR } \rightarrow \text { LOSet } \\
& \text { LOSet }=\{\text { LO: LO } \in \text { LOMR AND } \\
& \mathrm{LO}_{\text {category }}=\text { Geral AND } \\
& \text { tema IN LO LOMFields[i] }\} \text {, }
\end{aligned}
$$

\subsubsection{Filtragem por Perfil de Aprendizagem}

A filtragem por perfil de aprendizagem objetiva buscar, entre os objetos relacionados ao tema, quais atendem ao perfil de aprendizagem de um determinado aluno, considerando as diferentes dimensões de preferências que caracterizam o perfil em questão.

Após a filtragem por conceitos, o sistema e-LORS inicia a filtragem por perfil de aprendizagem, a qual será realizada considerando somente o conjunto de referências obtidos como resultado na etapa anterior (LOSet). As preferências do aluno descritas em seu perfil de aprendizagem (Tabela 3) são comparadas com os campos Tipo de Interatividade e Tipo de Recurso de Aprendizagem

\begin{tabular}{|c|c|c|c|}
\hline $\begin{array}{c}\text { Campo } \\
\text { LOM }\end{array}$ & $\begin{array}{l}\text { Valor do campo } \\
\text { (Tabela 3) }\end{array}$ & $\begin{array}{l}\text { Característica do } \\
\text { Perfil (Tabela 1) }\end{array}$ & $\begin{array}{l}\text { Dimensão de } \\
\text { Preferência }\end{array}$ \\
\hline \multirow{2}{*}{ 을 } & Ativo & Sensorial & \multirow{2}{*}{ 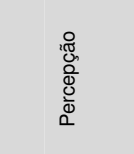 } \\
\hline & Expositivo & Intuitivo & \\
\hline
\end{tabular}
da categoria Educacional do padrão LOM (Tabela 4). A Tabela 5 apresenta o relacionamento entre as preferências e os campos do LOM adotados pela filtragem de perfil de aprendizagem.

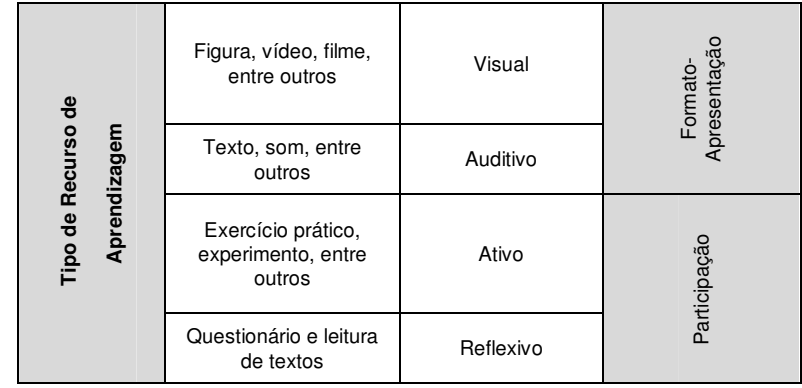

Tabela 5: Relacionamento entre campos do LOM e as dimensões de preferências.

O método searchLOLearningP irá selecionar os LOs, a partir do LOSet, que estejam em consonância com o perfil de aprendizagem do aluno. Ele irá produzir um novo conjunto, denominado LOSubset, como formalizado na equação (3) e no predicado (4).

$$
\text { searchLOLearningP: LOSet } \rightarrow \text { LOSubset }
$$

$$
\begin{aligned}
& \text { LOSubset }=\{\text { LO: LO } \in \text { LOSet AND (4) } \\
& \\
& \text { PreferenceCategory }[j] \exists \text { Modelo do Aluno AND } \\
& \text { PreferenceCategory }[\mathrm{j}] \leftrightarrow \text { LO }_{\text {LOMFields }[\mathrm{i}]} \\
&\}, \text { onde: } \\
& \mathrm{j}=\{\text { Percepção, Formato-Apresentação, } \\
&\text { Participação }\} \text { e } \\
& \mathrm{i}=\{\text { Interatividade, Recurso de Aprendizagem }\}
\end{aligned}
$$

Por exemplo, um aluno cuja categoria de preferência j = Percepção possui o valor Sensorial ; e cuja categoria j = Formato-Apresentação possui valor Visual, requer que os objetos sugeridos a este aluno devam possuir o valor Ativo no campo i = Tipo de Interatividade e um dos valores Figura, Vídeo, ou Filme no campo i = Tipo de Recurso de Aprendizagem. Assim como estabelecido na Tabela 5, lendo-se da direita para a esquerda.

\subsubsection{Filtragem por Tecnologia}

A última etapa do e-LORS é a filtragem dos LOs pela característica da tecnologia que o aluno está utilizando. Este item é opcional, conforme descrito anteriormente, pois pode não ser considerado no modelo do aluno. Seu objetivo é verificar questões tecnológicas que podem restringir a utilização de um LO como, por exemplo, o formato de um vídeo ou softwares necessários para que o LO possa ser utilizado.

A filtragem é realizada pelo método searchLOTech e utiliza o subconjunto obtido após a filtragem por perfil de aprendizagem (LOSubset) como entrada. Antes de iniciar a filtragem, o método verifica se existem restrições definidas no modelo do aluno; caso não existam, o novo subconjunto será igual ao encontrado na etapa anterior. $\mathrm{O}$ resultado será um novo subconjunto (LOSubsetF) que 
atenderá às questões tecnológicas existentes no modelo do aluno, assim como descrito pela equação (5) e pelo predicado (6).

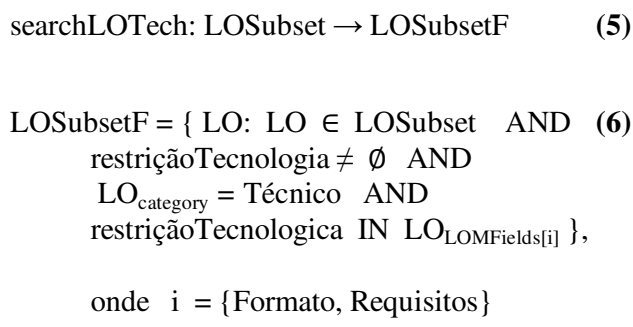

\section{Validação do Sistema e-LORS}

Um protótipo do sistema e-LORS foi desenvolvido e aplicado em um experimento realizado na disciplina de Física I para os cursos de Engenharia Civil, Engenharia da Computação e Engenharia Elétrica da Faculdade de Engenharia de Sorocaba (FACENS). O propósito do experimento era que os alunos utilizassem objetos de aprendizagem relacionados à área de Física como complemento das aulas presenciais.

Para a realização dessa experiência não foi adotado um gerenciador de cursos como o Moodle [24] ou o Tidia [34]. Ao invés disso, foi desenvolvido um sistema que controlasse o acesso dos usuários através de login e senha, permitindo o acompanhamento das atividades.

O sistema e-LORS foi desenvolvido como um componente Java para que pudesse ser integrado a diferentes ambientes de aprendizagem. Sua interface define os métodos de acesso, as funcionalidades de recomendação, e configuração para acesso ao repositório LOM, e ao modelo do aluno que será considerado.

Além disto, um repositório com os perfis do aluno foi planejado e elaborado de acordo com o modelo do aluno proposto (Tabela 3). Os objetos do repositório foram definidos de acordo com os campos LOM esperados pelo sistema (Tabela 4).

\subsection{Experimento}

Para o experimento foi escolhida a disciplina de Física I devido ao fato de que ela é aplicada em diferentes cursos da universidade, definindo assim uma diversidade considerável de alunos participando do experimento. Além disto, a área de Física possui muitos objetos de aprendizagem disponíveis gratuitamente na Web, possibilitando a elaboração de quantidade suficiente de objetos para atender aos diferentes perfis de aprendizagem. Participaram do experimento quatro professores, quatro assistentes de docência, e 297 alunos matriculados na disciplina de Física I dos cursos de Engenharia da Computação, Engenharia Civil, e Engenharia Elétrica. Esta equipe teria como tarefa a elaboração de objetos de aprendizagem de acordo com os conteúdos do curso de Física I.

A partir disto, seguiram-se as seguintes etapas: identificação dos perfis de aprendizagem dos alunos, catalogação de objetos de aprendizagem, execução do experimento com os alunos, e análise dos resultados do experimento.

\subsubsection{Identificação do Perfil}

Um questionário eletrônico foi aplicado aos alunos no momento da matrícula semestral; neste questionário os alunos declararam suas preferências de aprendizagem. O questionário foi baseado no trabalho de Soloman e Felder [6] que reflete a proposta de Felder e Silverman de estilos de aprendizagem [29].

Com base nas respostas, foram catalogadas as preferências previstas pelo modelo de aluno para cada estudante. Observou-se a ocorrência de cinco tipos de perfis, conforme listado na Tabela 6.

\begin{tabular}{|c|c|c|c|}
\cline { 2 - 4 } \multicolumn{1}{c|}{} & \multicolumn{3}{c|}{ Dimensões } \\
\hline Perfil & Percepção & Formato-Apresentação & Participação \\
\hline A & Intuitivo & Verbal & Reflexivo \\
\hline B & Intuitivo & Verbal & Ativo \\
\hline C & Sensorial & Verbal & Ativo \\
\hline D & Sensorial & Visual & Ativo \\
\hline E & Sensorial & Verbal & Reflexivo \\
\hline
\end{tabular}

Tabela 6: Perfis identificados a partir da aplicação do questionário.

A Figura 2 apresenta um gráfico da distribuição dos perfis. Observa-se que houve uma maior ocorrência do perfil Sensorial-Visual-Ativo, $49 \%$. 


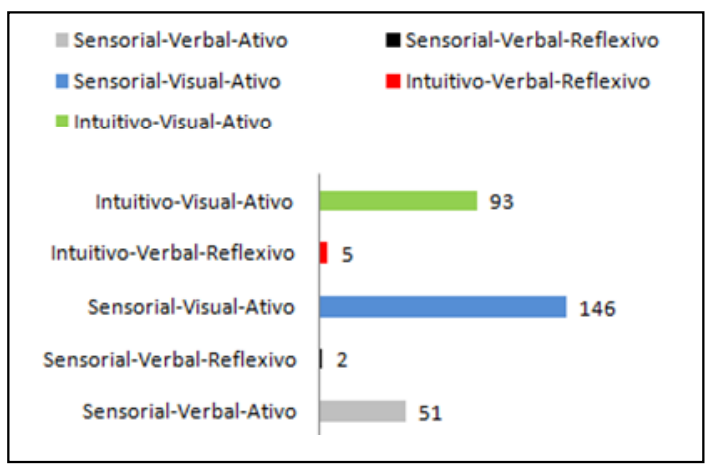

Figura 2: Distribuição dos perfis identificados.

Verificando-se a distribuição por dimensão de preferências, observou-se que há uma predominância dos perfis Visual e Ativo para as categorias FormatoApresentação e Participação do aluno, respectivamente. Isto evidencia a tendência por métodos de ensino ativos e visuais, segundo os quais devem ser oferecidas atividades que permitam a participação do aluno como fóruns, e aplicativos online; e que contenham imagens, e vídeos. Os docentes envolvidos na experiência acreditam que os alunos de Física I necessitam de métodos de ensino visuais, porque essa é uma disciplina que está no início do curso, quando o aluno ainda tem dificuldades em trabalhar com questões abstratas.

As informações pessoais dos estudantes foram importadas a partir de um cadastro já existente na instituição. $\mathrm{O}$ componente de tecnologia não foi considerado neste experimento.

\subsubsection{Elaboração e Catalogação dos LOs}

Para a experiência foram cadastrados diversos objetos de aprendizagem para a disciplina de Física I procurando abranger diferentes estilos de aprendizagem.

O grupo de professores e assistentes trabalharam no desenvolvimento e catalogação dos objetos. Os professores ficaram responsáveis por planejar os objetos de aprendizagem observando que tais objetos deveriam atender às especificidades do modelo proposto por Felder e Silverman. Os assistentes desenvolveram alguns objetos e adaptaram outros já existentes para que pudessem ser adotados nas disciplinas. Estes objetos foram catalogados segundo o padrão LOM em um repositório local da instituição.

Foram catalogados 100 objetos de aprendizagem correspondendo às sete diferentes lições de Física I. São exemplos de objetos cadastrados: simulações demonstrativas, simulações interativas, textos explicativos, figuras, e estudos de caso.

\subsubsection{Execução do Experimento}

A partir da atualização das categorias de preferências dos alunos, o sistema foi disponibilizado durante todo um semestre letivo. Neste período, 297 alunos da disciplina de Física I interagiram com o sistema de recomendação.

Em operação, o sistema e-LORS realizou as recomendações de conteúdo considerando as preferências identificadas durante a matrícula dos alunos e os objetos de aprendizagem catalogados no repositório LOM. O aluno entrava no tema de estudo e a partir disto o sistema de aprendizagem experimental enviava ao sistema e-LORS o tema objeto de estudo e a identificação do aluno que estava interagindo - iniciavam-se as etapas de filtragem.

Como exemplificação, suponha um aluno identificado como sendo do perfil D (Sensorial-Visual-Ativo) e que escolheu o tema Leis de Newton. Neste cenário, seguiramse os seguintes passos:

- Filtragem por conceitos: buscou e selecionou todas as ocorrências em que "Leis de Newton" aparecia nos campos da Categoria LOM Geral. Foram retornados 10 identificadores de objetos de aprendizagem diferentes. Este conjunto de objetos (LOSet) foi enviado à próxima etapa;

- Filtragem por perfil de aprendizagem: a partir do conjunto enviado (LOSet) esta etapa buscou e selecionou os objetos cujos metadados se encaixam no perfil D (Sensorial-Visual-Ativo). Este passo utilizou a categoria Educacional do padrão LOM e as categorias de preferências cadastradas para o aluno. Foram retornados dois objetos: um exercício prático com interação do aluno, e um exercício prático com apresentação de imagem;

- Filtragem por tecnologia: não foi considerada, pois o modelo do aluno não tratou este item.

Ao finalizar o processo de recomendação, o sistema retornou o conjunto de objetos selecionados e montou a visão para o aluno, permitindo que ele escolhesse o objeto com o qual desejasse interagir.

\subsubsection{Análise dos Resultados}

A implementação da arquitetura do sistema e-LORS demonstrou que a proposta de filtragem por etapas é uma engenharia promitente. Esta abordagem permitiu que diferentes necessidades da interação fossem atendidas: conceito, perfil de aprendizagem, e tecnologia. Para tanto, as preferências dos estudantes, descritas por meio de dimensões discretas, permitiram a automatização do processo de recomendação.

Ao final do experimento foi aplicado um questionário 
para medir a aceitação do sistema por parte dos alunos. Neste questionário, cada aluno deveria responder sobre seu grau de satisfação em relação aos objetos oferecidos, sendo 4 o mais alto grau de satisfação e 1 o mais baixo grau. O experimento demonstrou que $67 \%$ dos alunos de Física I consideraram as recomendações satisfatórias (notas 3 ou 4). Os alunos que demonstraram estar pouco satisfeitos ou insatisfeitos apontaram que gostariam de ter mais opções de objetos de aprendizagem para escolher. Observa-se que a maior parte dos alunos teve como recomendação objetos de aprendizagem como figuras, vídeos, simulações com interação, e estudos de caso.

Os docentes envolvidos no experimento relataram que houve uma participação ativa dos alunos da disciplina, e que os acessos aos materiais motivaram os alunos a interagirem mais, inclusive durante as aulas presenciais. Um aspecto negativo levantado pelos docentes e assistentes foi o tempo gasto para o desenvolvimento dos objetos de aprendizagem. Para as próximas edições os docentes e assistentes deverão construir mais objetos e aperfeiçoar os já existentes.

As médias obtidas pelos alunos que participaram da experiência foram importadas do sistema de controle acadêmico da instituição. Para os três cursos envolvidos no experimento, foram consideradas as médias dos alunos em dois grandes grupos. No primeiro grupo - o de controle - estavam os alunos que cursaram a disciplina no período anterior ao experimento, ou seja, que não utilizaram o sistema e-LORS. O segundo grupo era composto pelo alunos que cursaram a disciplina no período do experimento utilizando o sistema. Foi calculada a média geral dos alunos para os dois grupos; o primeiro grupo teve a média geral de 5,42 e o segundo de 7,55. Estes dados apontam para um significativo aumento de aproveitamento dos alunos que usaram o sistema de recomendação. Observa-se, todavia, que os dados se referem a grupos distintos e não à repetição do experimento por um mesmo grupo; o quê seria desejável, mas inviável, pois um mesmo grupo não cursaria o mesmo curso duas vezes. Como ressalva aos resultados, é fato que os resultados sofreram influência das características de cada turma um vestibular mais concorrido, por exemplo. Ainda assim, os relatos e a magnitude dos números sugere a validade das conclusões com significativa probabilidade.

\section{Trabalhos Relacionados}

A proposta deste trabalho foi baseada em trabalhos que abordavam a recomendação de conteúdos sobre a identificação de perfis de aprendizagem [8] [10][15][18].

Dentre estes trabalhos, Romero et al. [8] propõem um componente que sugere links para personalizar a interação do usuário baseado em algoritmos de mineração de dados que avaliam o $\log$ do estudante. O componente foi integrado ao projeto AHA! que é um ambiente de aprendizagem eletrônica que adota os conceitos de hipermídia adaptativa para o oferecimento de conteúdo aos alunos. As recomendações são realizadas com base em links cadastrados para o curso. Porém, a relação entre os objetos de aprendizagem e os perfis não é realizada de maneira automatizada, sendo necessário que o docente realize a correlação manualmente no momento do cadastro dos objetos.

O trabalho apresentado por Milošević et al. [10] constrói cenários de aprendizagem de acordo com o modelo de Kolb, que relaciona o estilo de aprendizagem com conteúdos de aprendizagem por meio do padrão SCORM (Sharable Content Object Reference Model) [5]. Embora trabalhe com padrões para especificação de conteúdos, a proposta não faz a associação do perfil de aprendizagem usando categorização de elementos.

O SEDHI (Sistema Educacional Hipermídia) [15] foi projetado para ser usado em cursos à distância na Web. Para tanto, ele realiza a recomendação de conteúdos com base no perfil do estudante na dimensão de seu conhecimento (iniciante, intermediário, ou avançado). O sistema realiza um acompanhamento das atividades realizadas pelo aluno e, com base nestas, e em seu perfil, classifica o estudante. É a partir desta classificação que o sistema realiza a adaptação da navegação no curso empregando as técnicas de ocultamento e anotação de links. Não há correlação automática entre os links e o perfil de aprendizagem.

O PAP (Política de Aprendizagem Personalizada) [18] define um conjunto de regras e procedimentos que permitem organizar de maneira flexível as atividades que serão apresentadas aos alunos durante a aprendizagem eletrônica. As regras devem ser especificadas estabelecendo-se condições associadas a eventos que podem ocorrer durante uma experiência de aprendizagem e que podem estar relacionadas a uma característica observável do aprendiz que comporá o seu perfil. A proposta não realiza uma vinculação dinâmica entre os conteúdos e as características do estilo de aprendizagem.

Silva, Mendes Neto e Jácome Júnior (2011) [38] apresentam uma proposta para recomendação de objetos de aprendizagem de acordo com o contexto de interação do usuário para dispositivos móveis. Os elementos de contexto considerado versam sobre informações do usuário e informações sobre localização geográfica entre outras. Através de multiagentes a arquitetura proposta realiza a recomendação de objetos de aprendizagem realizando a filtragem baseada em conteúdos e a filtragem baseada em contexto. 


\section{Considerações Finais}

Este trabalho propôs o sistema e-LORS, uma abordagem para a recomendação de objetos de aprendizagem considerando o perfil de aprendizagem do aluno. O sistema proposto possibilitou realizar a associação entre objetos e perfis de aprendizagem de maneira automatizada. Como objetivo, buscou-se apresentar o perfil de aprendizagem sob diferentes perspectivas como forma de relacionar as preferências sob diferentes aspectos. Para isto foi adotado o modelo de estilos de aprendizagem proposto por Felder e Silverman na especificação das preferências que seriam consideradas no perfil de aprendizagem do aluno.

Outro ponto importante foi a determinação da relação entre as preferências definidas no modelo do aluno com as categorias descritoras dos objetos de aprendizagem do padrão LOM. Com o uso do modelo de perfil de Felder e Silverman, foi proposto o perfil de aprendizagem do aluno. A adoção do padrão LOM, cujas descrições categóricas correspondem às dimensões de perfil adotadas nesse trabalho, permitiu a criação dinâmica de relacionamentos com os métodos de ensino correspondentes às diversas preferências.

Outro ponto da proposta é a segmentação da filtragem de conteúdo para recomendação, atribuindo diferentes responsabilidades às etapas: conceito, perfil de aprendizagem, e tecnologia. Além de permitir que a filtragem seja realizada em etapas bem definidas, a segmentação permite que a recuperação dos objetos possa ser, futuramente, estendida contemplando outros aspectos relevantes.

A abordagem proposta foi aplicada em um experimento com os alunos dos cursos de Engenharia Civil, Engenharia da Computação, e Engenharia Elétrica na disciplina de Física I durante um semestre letivo. A experiência teve como objetivo apoiar as atividades realizadas por meio de ensino presencial, procurando estimular os alunos a estudarem usando diferentes objetos de aprendizagem. Observou-se que o sistema e-LORS permitiu empregar uma automatização importante por meio do relacionamento do perfil e do tipo de objeto de aprendizagem. As fases de recomendação foram executadas cumprindo os objetivos da proposta e-LORS.

Como trabalhos futuros, citam-se as seguintes possibilidades: adaptação do perfil dos usuários baseada no uso dos objetos de aprendizagem, aprendizagem em ambiente ubíquo, ranqueamento dos objetos retornados pelo sistema e-LORs, avaliação de uso do sistema em plataformas com limitações de tamanho, processamento e interação, e o desenvolvimento de uma versão do sistema usando tecnologia Web 2.0, segundo a qual os alunos poderiam criar conteúdo.

\section{Referências}

[1] A. Felfernig, G. Friedrich, L. Schmidt-Thieme Recommender Systems. In: IEEE Intelligent Systems. 22 (3): 18-21, 2007.

[2] A. A. S. Richmond, R. R. Cummings. Implementing Kolb's learning styles into online distance ed1ucation. In: International Journal of Technology in Teaching and Learning. 1(1): 4554, 2005.

[3] A. Kobsa. Generic User Modeling System. In P. Brusilovsky, A. Kobsa, W. Nejdl (eds.) The Adaptive Web. LNCS 4321: 136-154, 2007.

[4] A. R. Vazquez, Y. A. Ostrovskaya. Analysis of Open Technological Standards for Learning Objects. In Proceedings of 4th LA-Web, Puebla Cholula, Mexico, 2006.

[5] ADL - SCORM. Disponível em: http://www.adlnet.gov/Technologies/scorm/defa ult.aspx. Acessado em: 21/08/2009.

[6] B. A. Soloman, R. M. Felder. Index of Learning Styles Questionnaire. Disponível em: http://www.engr.ncsu.edu/learningstyles/ilsweb.h tml. Acessado em: 02/04/2008.

[7] C. Lowery. Adapting to Student Learning Styles in a First Year Electrical/Electronic Engineering Degree Module. In Journal of the Higher Education Academy Engineering Subject Centre, 4(1): 52-60, 2009.

Disponível em: http://www.engsc.ac.uk/journal/index.php/ee/arti cle/view/80/153. Acessado em: 04/08/2009.

[8] C. Romero, S. Ventura, J. A. Delgado, P. De Bra. Personalized Links Recommendation Based on Data Mining in Adaptive Educational Hypermedia Systems. In: Creating New Learning Experiences on a Global Scale, Springer LNCS 4753: 292-306, Crete, 2007.

[9] C. Soles, L. Moller. Myers Briggs Type Preferences in Distance Learning Education. In International Journal of Educational Technology, 2(2), 2001.

[10] D. Milošević, M. Brković, M. Debevc, R. Krneta. Adaptive Learning by Using SCOs Metadata. Interdisciplinary Journal of Knowledge and Learning Objects, Vol. 3, 2007.

[11] E. Lumsdaine, H. A. Evensen, P. F. Zenner. Educating Designers on Design via Distance Learning. In: Proceedings of the 2005 American 
Society for Engineering Education Annual Conference \& Exposition, 2005.

[12] F. Coffield et al . Learning styles and pedagogy in post-16 learning. A systematic and critical review. Learning and Skills Research Centre. London, 2004.

[13] F. Coffield, D. Moseley, E. Hall, K. Ecclestone. Learning styles and pedagogy in post-16 learning. A systematic and critical review. In: Learning and Skills Research Centre, 2004.

[14] G. D. Magoulas, K. Papanikolaou, M. Grigoriadou. Adaptive web-based learning: accommodating individual differences through system's adaptation. In: British Journal of Educational Technology. 34(4): 511-527, United Kington, 2003.

[15] G. T. Silva, M. C. Rosatelli. Adaptation in Educational Hypermedia based on the Classification of the User Profile. In Proceedings of the 9th International Conference on Intelligent Tutoring Systems (ITS 2006), pp. 268-277, 2006.

[16] A. Gomes, A. Santos, L. Carmo, A. J. Mendes. Learning styles in an e-learning tool. In Proceeding of International Conference on Engineering Education, ICEE 2007, Portugal, 2007.

[17] H. Lu, L. Jia, S. S. Gong, B. B. Clark. The Relationship of Kolb Learning Styles, Online Learning Behaviors and Learning Outcomes. In Educational Technology \& Society, 10(4): 187-196, 2007.

[18] I. Stiubiener, M. C. Rosatelli, W. V. Ruggiero. An approach to personalisation in e-learning. In Proceeding of IEEE International Conference on Advanced Learning Technologies, Japão, 2007.

[19] IEEE LOM. Draft standard for learning object metadata. 2002. Disponível em: http://ltsc.ieee.org/wg12/index.html. Acesso: 13/08/2009.

[20] L. A. M. Zaina, G. Bressan. Classification of learning profile based on categories of student preferences. In Proceedings 38th Annual Frontiers in Education Conference, pp. F4E-1-F4E-6, 2008.

[21] L. A. M. Zaina, G. Bressan.Learning objects retrieval from contextual analysis of user preferences to enhance e-learning personalization. In: Proceedings of IADIS International Conference WWW/Internet 2009, pp. 237-244, 2009.

[22] M. Bilgic, R. J. Mooney. Explaining Recommendations: Satisfaction vs. Promotion. Work- shop: Beyond Personalization 2005.IUI'05, San Diego, California, USA, 2005.

[23] M. J. Pazzani, D. Billsus. Content-Based Recommendation Systems. In The Adaptive Web, Brusilovsky, P., Kobsa, A., and Nejdl, W. (Eds.). 4321: 325-341, 2007.

[24] Moodle. Disponível em: http://moodle.org/. Acessado em: 21/08/2009.

[25] N. Salim, N. Haro. The Construction of Fuzzy Set and Fuzzy Rule for Mixed Approach in Adaptive Hypermedia Learning System. In: Technologies for E-Learning and Digital Entertainment. Lecture Notes in Computer Science, 3942:183-187, 2006.

[26] N. Stash, A. Cristea, P. De Bra. Authoring of Learning Styles in Adaptive Hypermedia: Problems and Solutions. In: Proceedings of WWW 2004, May 17-22, New York, pp. 114-123, 2004.

[27] P. Brusilovsky, E. Millán. User Models for Adaptive Hypermedia and Adaptive Educational System. In P. Brusilovsky, A. Kobsa, W. Nejdl (eds.) The Adaptive Web. LNCS 4321: 54 - 89, 2007.

[28] R. Burke. Hybrid Recommender Systems: Survey and Experiments. User Modeling and User-Adapted Interaction. 12(4): 331-370, 2002.

[29] R. M. Felder, K. L. Silverman. Learning and Teaching Styles in Engineering Education. Journal of Engineering Education. 78 (7): 674-681, 1988.

[30] R. M. Felder, R. Brent, Understanding Student Differences, Journal of Engineering Education, vol. 94, no. 1, pp 57-72, 2005.

[31] R. McGreal. Learning Objects: A Practical definition. International Journal of Instructional Technology and Distance Learning. 1(9), 2004.

[32] S. Graf, Kinshuk, T-C. Liu. Identifying Learning Styles in Learning Management Systems by Using Indications from Students' Behaviour. In: Proceedings of the 2008 Eighth IEEE International Conference on Advanced Learning Technologies, pp. 482-486, 2008.

[33] S. Graf, S. R. Viola, Kinshuk, T. Leo. In-depth Analysis of the Felder-Silverman Learning Style Dimensions. Journal of Research on Technology in Education, 40(1):79-93, 2007. 
[34] Tidia-Ae. Disponível em: http://tidiaae.usp.br/portal. Acessado em: 21/08/2009.

[35] U. Hanani, B. Shapira, P. Shoval. Information Filtering: Overview of Issues, Research and Systems. In: User Modeling and User-Adapted Interaction. 11(3): 203-259, 2004.

[36] V. Devedžić, D. Gašević, D. Djurić. Clarifying the meta. In International Journal of Information and Communication Technology, 1(2):148-158, 2008.

[37] Y. Wang, N. Stash, L. Aroyo, L. Hollink, G. Schreiber. Semantic Relations in Content-based Recommender. In Proc. of the Fifth International Conference on Knowledge Capture, 209-210, 2009.

[38] L. C. N. da Silva, F. M. Mendes Neto, L. Jácome Júnior. MobiLE: Um ambiente Multiagente de Aprendizagem Móvel para Apoiar a Recomendação Sensível ao Contexto de Objetos de Aprendizagem. In Proc. XXII Simpósio Brasileiro de Informática na Educação e XVII Workshop de Informática na Educação, 254-263, Aracajú, 2011.

[39] V. Bremgartner, J. F. M. Netto. Auxílio Personalizado a Estudantes em Ambientes Virtuais de Aprendizagem Utilizando Agentes e Competências. In Proc. XXII Simpósio Brasileiro de Informática na Educação e XVII Workshop de Informática na Educação, 40-49, Aracajú, 2011.

[40] L. L. Lazzarotto, J. L. Braga, A. P. Oliveira, F. J. V. Passos. A educação em ambientes virtuais: proposição de recursos computacionais para aumentar a eficiência do processo ensinoaprendizagem. In: Revista Brasileira de Informática na Educação, 19(2):42-55, 2011. 